Zhao Ling, Wang Xin, Yang Liguo* and Gao Linna

\title{
Crystal structure of ((5,5'-dimethoxy-2, $2^{\prime}-(1,2-$ phenylenebis(nitrilomethylidyne)))diphenolato- $\left.\mathrm{K}^{4} \mathrm{O}, \mathrm{N}, \mathrm{O}^{\prime}, \mathrm{N}^{\prime}\right)$ copper(II), $\mathrm{C}_{22} \mathrm{H}_{18} \mathrm{~N}_{2} \mathrm{CuO}_{4}$
}

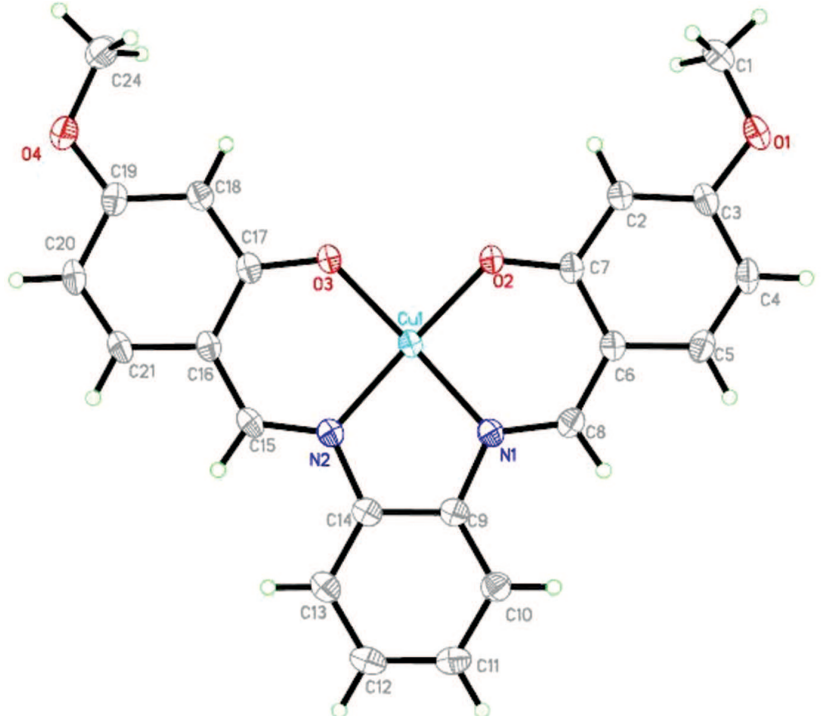

https://doi.org/10.1515/ncrs-2018-0361

Received September 8, 2018; accepted November 12, 2018;

available online December 5, 2018

\section{Abstract} $b=5.3211(4) \AA, \quad c=21.6563(16) \AA, \quad \beta=92.129(4)^{\circ}$, $V=1817.9(2) \AA^{3}, Z=4, \quad R_{\mathrm{gt}}(F)=0.0254, \quad w R_{\mathrm{ref}}\left(F^{2}\right)=0.0765$, $T=298 \mathrm{~K}$.

\section{CCDC no.: 1878573}

The crystal structure is shown in the figure. Tables 1 and 2 contain details on crystal structure and measurement conditions and a list of the atoms including atomic coordinates and displacement parameters.

*Corresponding author: Yang Liguo, College of Chemistry and Environmental Engineering, Anyang Institute of Technology, Anyang 455000, Henan, P.R. China, e-mail: lgyang@ayit.edu.cn

Zhao Ling and Wang Xin: College of Chemistry and Environmental Engineering, Anyang Institute of Technology, Anyang 455000, Henan, P.R. China

Gao Linna: College of Chemical and Environmental Engineering, Shandong University of Science and Technology, Qingdao 266590 , P.R. China
$\mathrm{C}_{22} \mathrm{H}_{18} \mathrm{~N}_{2} \mathrm{CuO}_{4}$, monoclinic, $P 2{ }_{1} / n$ (no. 14), $a=15.7861$ (11) $\AA$,

Table 1: Data collection and handling.

\begin{tabular}{ll}
\hline Crystal: & Blue block \\
Size: & $0.12 \times 0.10 \times 0.08 \mathrm{~mm}$ \\
Wavelength: & Mo $K \alpha$ radiation $(0.71073 \AA$ A $)$ \\
$\mu:$ & $1.24 \mathrm{~mm}^{-1}$ \\
Diffractometer, scan mode: & APEX2, $\varphi$ and $\omega$ \\
$\theta_{\text {max }}$, completeness: & $25.0^{\circ},>99 \%$ \\
$N\left(h k l_{\text {measured }}, N(h k l)_{\text {unique }}, R_{\text {int }}:\right.$ & $24774,3205,0.029$ \\
Criterion for $I_{\text {obs }}, N(h k l)_{\mathrm{gt}}:$ & $I_{\text {obs }}>2 \sigma\left(I_{\text {obs }}\right), 2888$ \\
$N(\text { param })_{\text {refined }}:$ & 264 \\
Programs: & Bruker $[1]$, SHELX [2, 3] \\
\hline
\end{tabular}

Table 2: Fractional atomic coordinates and isotropic or equivalent isotropic displacement parameters $\left(\AA^{2}\right)$.

\begin{tabular}{lrrrr}
\hline Atom & $\boldsymbol{x}$ & $\boldsymbol{y}$ & $\boldsymbol{z}$ & $\boldsymbol{U}_{\text {iso }}{ }^{*} \boldsymbol{U}_{\text {eq }}$ \\
\hline Cu1 & $0.55216(2)$ & $0.70941(5)$ & $0.66651(2)$ & $0.03281(10)$ \\
N1 & $0.59322(10)$ & $0.9898(3)$ & $0.71656(7)$ & $0.0324(4)$ \\
N2 & $0.46405(10)$ & $0.6937(3)$ & $0.72637(7)$ & $0.0321(4)$ \\
O1 & $0.88902(10)$ & $1.0285(3)$ & $0.51769(7)$ & $0.0503(4)$ \\
O2 & $0.64259(10)$ & $0.7268(3)$ & $0.61170(7)$ & $0.0478(4)$ \\
O3 & $0.51050(9)$ & $0.4293(3)$ & $0.62042(6)$ & $0.0395(3)$ \\
O4 & $0.32969(11)$ & $-0.2400(3)$ & $0.55813(8)$ & $0.0490(4)$ \\
C1 & $0.88868(18)$ & $0.8170(5)$ & $0.47759(12)$ & $0.0603(7)$ \\
H1A & 0.8878 & 0.6655 & 0.5016 & $0.090^{*}$ \\
H1B & 0.9387 & 0.8196 & 0.4537 & $0.090^{*}$ \\
H1C & 0.8393 & 0.8231 & 0.4503 & $0.090^{*}$ \\
C2 & $0.76391(13)$ & $0.8677(4)$ & $0.56504(10)$ & $0.0393(5)$ \\
H2 & 0.7596 & 0.7358 & 0.5369 & $0.047^{*}$ \\
C3 & $0.82867(13)$ & $1.0382(4)$ & $0.56081(9)$ & $0.0382(5)$ \\
C4 & $0.83739(15)$ & $1.2385(4)$ & $0.60250(11)$ & $0.0448(5)$ \\
H4 & 0.8811 & 1.3542 & 0.5991 & $0.054^{*}$ \\
C5 & $0.78127(15)$ & $1.2609(4)$ & $0.64774(11)$ & $0.0436(5)$ \\
H5 & 0.7873 & 1.3939 & 0.6754 & $0.052^{*}$ \\
C6 & $0.71327(12)$ & $1.0892(4)$ & $0.65473(9)$ & $0.0347(4)$ \\
C7 & $0.70367(12)$ & $0.8893(4)$ & $0.61146(9)$ & $0.0353(4)$ \\
C8 & $0.65797(13)$ & $1.1290(4)$ & $0.70358(9)$ & $0.0364(4)$ \\
H8 & 0.6688 & 1.2676 & 0.7288 & $0.044^{*}$ \\
C9 & $0.54211(12)$ & $1.0421(4)$ & $0.76736(9)$ & $0.0334(4)$ \\
C10 & $0.55541(14)$ & $1.2327(4)$ & $0.81004(10)$ & $0.0398(5)$ \\
H10 & 0.6019 & 1.3386 & 0.8070 & $0.048^{*}$ \\
C11 & $0.49993(15)$ & $1.2664(4)$ & $0.85712(11)$ & $0.0450(5)$ \\
H11 & 0.5091 & 1.3956 & 0.8855 & $0.054^{*}$ \\
C12 & $0.43101(15)$ & $1.1096(5)$ & $0.86228(10)$ & $0.0472(5)$ \\
& & & &
\end{tabular}

ð Open Access. ( 2019 Zhao Ling et al., published by De Gruyter. (c) BY This work is licensed under the Creative Commons Attribution 4.0 Public License. 
Table 2 (continued)

\begin{tabular}{lrrrr}
\hline Atom & $\boldsymbol{x}$ & $\boldsymbol{y}$ & $\boldsymbol{z}$ & $\boldsymbol{U}_{\text {iso }} \boldsymbol{U}_{\text {eq }}$ \\
\hline H12 & 0.3935 & 1.1343 & 0.8939 & $0.057^{*}$ \\
$\mathrm{C} 13$ & $0.41753(14)$ & $0.9165(4)$ & $0.82080(10)$ & $0.0429(5)$ \\
H13 & 0.3715 & 0.8097 & 0.8250 & $0.051^{*}$ \\
$\mathrm{C} 14$ & $0.47201(12)$ & $0.8793(4)$ & $0.77270(9)$ & $0.0338(4)$ \\
$\mathrm{C} 15$ & $0.40166(12)$ & $0.5323(4)$ & $0.72438(9)$ & $0.0349(4)$ \\
H15 & 0.3624 & 0.5443 & 0.7552 & $0.042^{*}$ \\
$\mathrm{C} 16$ & $0.38841(12)$ & $0.3424(4)$ & $0.68003(9)$ & $0.0333(4)$ \\
$\mathrm{C} 17$ & $0.44322(12)$ & $0.2969(4)$ & $0.63026(9)$ & $0.0337(4)$ \\
$\mathrm{C} 18$ & $0.42381(13)$ & $0.0969(4)$ & $0.58957(9)$ & $0.0380(5)$ \\
H18 & 0.4600 & 0.0630 & 0.5577 & $0.046^{*}$ \\
C19 & $0.35265(13)$ & $-0.0498(4)$ & $0.59576(9)$ & $0.0384(5)$ \\
C20 & $0.29804(13)$ & $-0.0041(5)$ & $0.64504(10)$ & $0.0435(5)$ \\
H20 & 0.2501 & -0.1027 & 0.6496 & $0.052^{*}$ \\
C21 & $0.31625(13)$ & $0.1839(4)$ & $0.68523(10)$ & $0.0404(5)$ \\
H21 & 0.2803 & 0.2111 & 0.7176 & $0.048^{*}$ \\
C24 & $0.38329(17)$ & $-0.2969(5)$ & $0.50765(11)$ & $0.0518(6)$ \\
H24A & 0.3870 & -0.1525 & 0.4813 & $0.078^{*}$ \\
H24B & 0.3598 & -0.4354 & 0.4844 & $0.078^{*}$ \\
H24C & 0.4389 & -0.3406 & 0.5236 & $0.078^{*}$ \\
\hline
\end{tabular}

\section{Source of material}

The educt, 6,6'-((1,2-phenylenebis(azanylylidene))bis (methanylylidene))bis(3-methoxyphenol) ligand was purchased from Acros Ltd. and used without further purification, the other reagents were commercially available and used as purchased [1-3]. A mixture of $\mathrm{CuCl}_{2}(53.78 \mathrm{mg}, 0.4 \mathrm{mmol})$, Schiff base ligand $(49.64 \mathrm{mg}, 0.4 \mathrm{mmol})$ and distilled water $(10 \mathrm{~mL})$ was sealed in a $23 \mathrm{~mL}$ Teflon-lined steel vessel (solution $\mathrm{pH} 2.5$ ) and heated at $160{ }^{\circ} \mathrm{C}$ for $72 \mathrm{~h}$, and then cooled to room temperature at a rate of $0.05{ }^{\circ} \mathrm{C} \mathrm{min}^{-1}$. The resulting light turquoise block crystals of the compound were obtained and washed with distilled water. The yield is ca. $32 \%$. IR( $\mathrm{KBr})$ : 3256(s), 1597(s), 1518(s), 1497(s), 1448(s), 1356(s), 1245(s), 1174(s), 1113(s), 1073(s), 1005(s), 924(s), 894(s), 835(m), 773(m), 716(m), 619(m), 493(m) $\mathrm{cm}^{-1}$.

\section{Experimental details}

All $\mathrm{H}$ atoms were placed geometrically and treated as riding on their parent atoms, with $\mathrm{C}-\mathrm{H}$ 0.96, with $U_{\text {iso }}(\mathrm{H})=1.5 U_{\text {eq }}(\mathrm{C})$.

\section{Comment}

There has been considerable interest in Schiff base ligands and their metal complexes due to their variety of applications in chemistry, biology, physics and in catalysis [4-6].
The compound crystallizes in the monoclinic space group $P 2_{1} / n$ with four formula units in the unit cell. As shown in the figure, one formula unit consists of one Schiff base ligand and one $\mathrm{Cu}$ atom. The $\mathrm{Cu} 1$ ion is in a slightly distorted square coordination environment, with two nitrogen atoms and two oxygen atoms from the Schiff base title ligand. The bond length of Cu1-N1 and Cu1-N2 is $1.939 \AA$ and $1.936 \AA$, while the bond length of $\mathrm{Cu} 1-\mathrm{O} 2$ and $\mathrm{Cu}-\mathrm{O} 3$ is $1.893 \AA$ and $1.899 \AA$. The geometric parameters are all in the expected ranges for such complexes [7-9].

Acknowledgements: This work was supported by Henan Science and Technology Project (Nos. 172102210161, 172102310166), the Key Scientific Research Projects of Colleges and Universities, Henan Province (No. 18A150020) and the Research Fund of Anyang Institute of Technology (No. YJJ2016014).

\section{References}

1. Bruker. APEX2, SAINT and SADABS. Bruker AXS Inc., Madison, WI, USA (2005).

2. Sheldrick, G. M.: SHELXT - integrated space-group and crystal-structure determination. Acta Crystallogr. A71 (2015) 3-8.

3. Sheldrick, G. M.: Crystal structure refinement with SHELXL. Acta Crystallogr. C71 (2015) 3-8.

4. Raisanen, M. T.; Nieger, M.; Slawin, A. M. Z.; Leskela, M.; Repo, T.: Two-and three-dimensional packing diagrams of $M$ (salophen) complexes. CrystEngComm 13 (2011) 4701-4708.

5. Funeriu, D. P.; Lehn, J. M.; Baum, G.; Fenske, D.: Double subroutine sel-assembly; spontaneous generation of a nanocyclic dodecanuclear $\mathrm{Cu}^{1}$ inorganic architecture. Chem. Eur. J. 3 (1997) 99-104.

6. Chen, C. T.; Suslick, K. S.: One-dimensional coordination polymers: application to matrial science. Coord. Chem. Rev. 128 (1993) 293-322.

7. Eltayeb, N. E.; Teoh, S. G.; Chantrapromma, S.; Fun, H. K.; Adnan, R.: Chlorido\{5,5'-dimethoxy-2,20-[1,2-phenylenebis (nitrilomethylidyne)]-diphenolato- $\left.{ }^{4} O, N, O^{\prime}, N^{\prime}\right\}$ manganese(III). Acta Crystallogr. E64 (2008) m670-m671.

8. Niu, M. J.; Fan, S. M.; Liu, K.; Cao, Z. Q.; Wang, D. Q.: [5, $5^{\prime}$-Dihydroxy-2,2'-[0-phenylenebis(nitrilomethylidyne)] diphenolato\}copper(II) methanol disolvatel. Acta Crystallogr. E66 (2010) m77.

9. Suresh, E.; Bhadbhade, M. M.; Srinivas, D.: Molecular association, chelate conformation and reactivity correlations in substituted o-phenylenebis(salicylidenato)copper(II) complexes: UV-visible, EPR and X-ray structural investigations. Polyhedron 15 (1996) 4133-4144. 